Document downloaded from:

http://hdl.handle.net/10251/103627

This paper must be cited as:

Climente Alarcon, V.; Nair, D.; Sundaria, R.; Antonino-Daviu, J.; Arkkio, A. (2017).

Combined Model for Simulating the Effect of Transients on a Damaged Rotor Cage. IEEE Transactions on Industry Applications. 53(4):3528-3537. doi:10.1109/TIA.2017.2691001

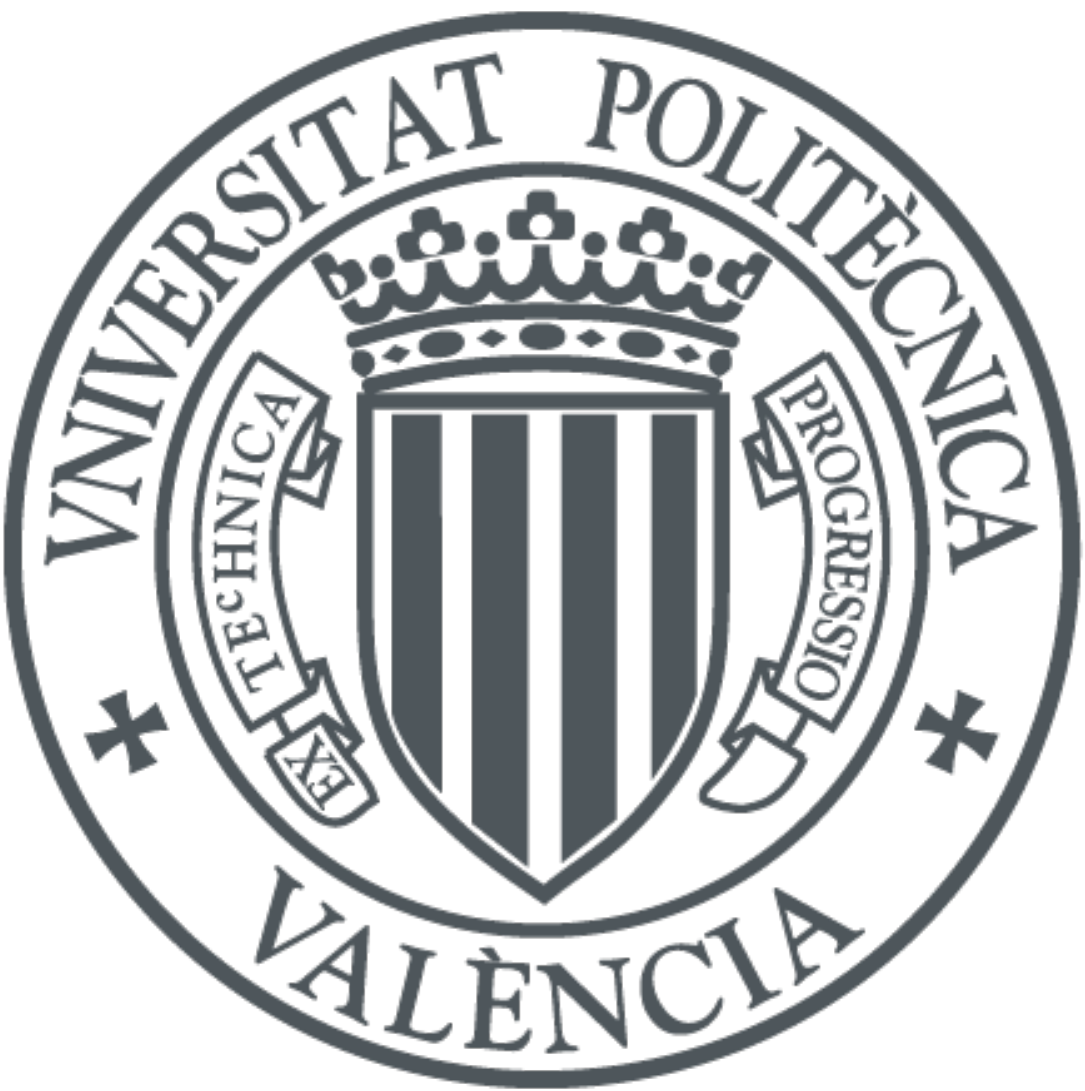

The final publication is available at

http://doi.org/10.1109/TIA.2017.2691001

Copyright Institute of Electrical and Electronics Engineers

Additional Information 


\title{
Combined Model for Simulating the Effect of Transients on a Damaged Rotor Cage
}

\author{
Vicente Climente-Alarcon, Devi Nair, Ravi Sundaria, Jose A. Antonino-Daviu, Antero Arkkio
}

\begin{abstract}
The expansion of the transient operation of electrical machines as, for instance, in vehicle traction applications, demands an accurate computation of the thermal behavior under these conditions in order to enhance the economy of the design and provide a precise estimation of the overload capacity. In addition, heavy transients have been identified as specially damaging for the rotor cage of large induction motors. The aim of this work is the development of a model able to simulate in detail the thermal and mechanical effects of a heavy transient on an induction's motor rotor featuring a damaged (with a reduced section on one of its ends) rotor bar. Some preliminary results that provide a qualitative understanding of the development of a bar breakage during a fatigue test are presented.
\end{abstract}

Index Terms-- Induction motors, prognostics and health management, rotors, finite element analysis, thermal stresses, transient analysis.

\section{NOMENCLATURE}

$B$ Peak magnetic flux density

$C$ Elastic modulus tensor

$C_{e}$ Thermal capacitance of an element

$C p$ Heat capacity

$\vec{d}$ Displacement field

$\vec{D}$ Electric flux density

$E$ Young modulus

$\vec{E} \quad$ Electric field

$f$ Supply frequency

$f_{s} \quad$ Supply frequency times slip

$G$ Thermal conductance

$h$ Heating power

$\vec{H}$ Magnetic field

I Current

$\vec{J}$ Current density

$k$ Thermal conductivity

$k_{h}, k_{e}, k_{a}$ Iron losses model's coefficients

$m_{\text {iron }}$ rotor's iron mass

$P \quad$ Generated power in an element

$q$ Thermal flux density

$R$ Resistance

S Slip

$\mathrm{t}$ Time

$\vec{u} \quad$ Unitary vector

$\alpha$ Iron losses model's exponent

V. Climente-Alarcon, D. Nair, R. Sundaria and A. Arkkio are with Department of Electrical Engineering and Automation, Aalto University, P.O. 13000, Espoo 00076, FINLAND (e-mails: viclial@ieee.org, devi.nair@aalto.fi, ravi.sundaria@aalto.fi, antero.arkkio@aalto.fi).

J. A. Antonino-Daviu is with the Instituto Tecnológico de la Energía, ITE, Universitat Politècnica de València, Camino de Vera s/n, 46022, Valencia, SPAIN (e-mail: joanda@die.upv.es).

\author{
$\beta$ Thermal expansion tensor \\ $\varepsilon \quad$ Linear strain \\ $\rho$ Charge density \\ $\rho_{d}$ Density \\ $\phi \quad$ Electric potential \\ $v$ Poisson ratio \\ $\sigma$ Electrical conductivity \\ $\tau$ Stress tensor \\ $\psi \quad$ Flux linkage \\ $\theta$ Temperature
}

\section{INTRODUCTION}

$\Upsilon^{\mathrm{i}}$ he power yielded by an electrical machine is limited by its maximum operating temperature. A more efficient cooling system or a colder environment allows the same machine to be operated above its rated power without decreasing the expected lifespan [1].

Small motors trust on internal air flow along the airgap, and external along its cover, to provide cooling. Although this approach is simple and needs no maintenance, it presents the drawback of linking the rotational speed of the machine to its cooling rate, whilst its heating remains proportional to the yielded torque. A balance could be achieved for nominal operation of motors connected to the grid; however, the introduction of variable speed drives (VSD) prevents following this approach any longer [2]. Furthermore, the use of these devices introduces harmonics in the supply voltage that completely deform the current waveform feeding the machine. These high frequency harmonics increase losses, and hence the heating rate of the motor [3].

Thus, for these cases a cost-effective solution is adding another electrical motor to independently move the fan. This arrangement allows providing an external air flow to increase cooling, although internally, along the airgap and around the end windings, heat transmission is not improved [4, 5]. Nevertheless, in several applications and motor configurations an independent cooling subsystem cannot be arranged. In vehicle in-wheel motors, for instance, the necessity of keeping the non-suspended weight as low as possible generally prevents the use of any additional elements. Moreover, for these tasks the geometry of the machine is completely altered, typically switching the positions of stator and rotor, now external to the first one. Also, in such dynamical applications [6] non-stationary operation prevails along the entire life cycle of the machine, therefore a thermal stationary state is never reached, and overload periods might be habitual, for instance, when starting the movement of the vehicle on slopes [7]. A careful thermal analysis during transient operation for this kind of 
applications is needed, since high temperatures have an immediate impact on this equipment due to their demagnetization effect [8].

Besides design, in which the intended objective is to maintain the maximum temperature below certain threshold to prevent reducing the lifespan of the machine, a novel application of thermal computation just lies in determining that lifespan when due to a defect, this limit is exceeded. Within the new Condition-Based Maintenance and Prognostics and Health Management (CBM/PHM) architecture, the output usually generated by diagnosis systems consisting of a binary value indicating the state of a machine or element (healthy/faulty) has evolved into a more detailed methodology by which the evolution of the fault is traced from its early stages by an array of advanced diagnosis methods [9] and extrapolated in order to predict the remaining useful life (RUL) of the equipment. The result of its application consists of a time-span value long enough to allow scheduling corrective actions [10].

Two kinds of approaches are followed for performing this prognosis of the defect: data-driven approach and the physics of failure (PoF) approach. The first one is specially employed when the mechanism of failure is not clear, whilst for the second one accurate thermal computations are essential since localized defects, such as hot spots in the stator windings, are the underlying cause of common failure modes in rotating electrical machines due to the cyclic stress that thermal expansion produces [11]. In this context, accurate thermal and mechanical modelling can provide an insight into the PoF that explain the growth and propagation of the defect, adding a complementary methodology to data-driven models for correctly predicting its evolution.

Rotor cage failures are faults well suited for the application of a full CBM/PHM system since they constitute a slow evolving defect and usually affect large machines started under high inertia [12], thus being equipment on which the deployment may be justified.

Despite the complexity required for the development of these models, which comprise several physical phenomena, there have appeared seminal studies on the matter since the beginning of the century. In [13], a comparison of aluminum and copper cages from the design point of view is presented, including their behavior during a direct-on-line startup, which is identified as the most demanding operating condition (especially under high inertia loads) and the connections of the bars to the end ring as the higher stress points. The conclusion is that copper is advantageous, especially from a thermal and mechanical point of view, due to its greater heat capacity and higher strength, although the underestimation of mechanical and thermal expansion stresses during the design stage and poor brazing during the manufacturing appear responsible in this case for premature failures [14]. Furthermore, it is also indicated that in a die cast cage any relative movement between bars and rings is not allowed, thus increasing stresses in the end-connectors joints compared to welded bar ones.

On the other hand, [15] tackled the problem directly by modelling and reproducing the fault itself. The actual equipment was analyzed through finite element analysis (FEA), followed by a series of fatigue tests. A time-stepping method was used to simulate a startup transient, being the tangential forces acting on the bars computed from the rotor acceleration. These values fed a mechanical FEM, in which the position of the nodes in the end rings was fixed, whilst the movement of the ones in the bars remained unrestricted. Even increasing the stresses in the end-connectors sections by adding the effect of cracks or other imperfections, it could not be reproduced a bar breakage. Fatigue testing on actual equipment yielded the same result, thus concluding this work that this fault is caused by a combination of both thermal and mechanical loads.

A third approach is carried out in [16] to explain actual damage observed in rotors. An analytical methodology is used to model the full electromagnetic-thermal-mechanical problem in order to obtain the stresses on the leakage filament joining the bars of two cages. This connection between the external, higher resistance cage for increasing the torque during the startup, and the internal one, intended to reduce their combined resistance during stationary operation, is needed to avoid the flux missing the deeper one and constitutes a weak point in the rotor's electrical circuit. Under severe operation, as it is usual in the harsh environment of the mining industry with frequent starting ups and stall conditions, the rising temperature in the leakage filament increases the stress of aluminum beyond its yield limit and when the rotor is allowed to cool down microfractures appear. If its melting point is exceeded, the result is a neat effect wherein the material flows out of the slot and projects across the airgap to finally damage the stator winding.

Ref. [17] provides a further insight of thermal aspects involved in the design of an induction motor. A per-phase analytical circuit and two thermal network models are used to study the heating of a healthy rotor cage in stationary operation, but also during transients such as startup and stall conditions. For these cases, the skin effect on the bars, which is modelled by a multi layered approach, and the heat transfer towards the rotor teeth are taken into account. The results are validated for the later transient by experimental tests on several rotors. An initial assessment of their mechanical effects in the lifespan of the machine is also performed.

Finally, the issue was fully addressed experimentally in [18] with the implementation of a fatigue test aimed at developing a bar breakage by subjecting an induction motor to heavy transients, in this case startup and plug stopping cycles. The results show that the fault could not be reproduced until the cage was weakened, basically creating a hot spot as studied in [16] but in this case at a bar's end. The effect of the fault's progression on the stator currents was fitted to a crack propagation model that allowed carrying out a prediction of the RUL of the bar once the fault had been detected at incipient level. Nevertheless, the requirements regarding a long, heavy cycling during the fatigue test imposed the selection of a small size machine, thus preventing the acquisition of the rotor magnitudes, as it is 
done in [17]. Therefore, although the driving mechanism behind the breakage was identified, a PoF model could not be established.

Thus the aim of this work is to develop a model capable of providing a further insight into the transient operation of damaged rotor cages by computing the rotor's electrical, thermal and mechanical magnitudes under which that breakage occurred. This model is improved and adjusted based on the measurements performed on the stator during the fatigue test presented in [18]. Given the complexity of the task, involving three areas of physics, several techniques are employed according to the degree of detail needed. Early studies in heat transfer [19] already advocated for FEM for assessing the effect of hot spots in an induction motor during transients. However, since this spatial accuracy is not needed in the stator, lumped parameters are preferred there, thus a thermal network is used to model this part of the machine $[20,21]$. Both FEM and lumped thermal models are linked by the power transferred through the Neumann boundary conditions set at the external surfaces of the rotor. This approach sharply reduces computational needs and simplifies the modelling of the convection in the air gap and end caps. Similarly, the rotor bars are loaded for each time step with the value of current computed by an electromagnetic (EM) analytical model in this case of the full machine, presented in [22], whilst losses and the skin effect are calculated from the rotational speed yielded by this EM model according to [23] for the former, whilst for the later a novel approach is utilized. In order to obtain the mechanical effects caused by thermal expansion, a post-processing stage computes the stresses and deformations according to the temperatures reached at the end of the transient.

The remainder of this paper is organized as follows, Section III is dedicated to introduce the calculation procedure followed as well as the FEM tool used, Section IV presents and initial validation according to the only available temperature sensor in the machine, Section $\mathrm{V}$ yields the results obtained and Section VI the corresponding conclusions.

\section{Methodology Followed}

The combined model used in this work is divided in two parts: the mesh depicting half of the rotor, on which the electrical, thermal and mechanical field variables are solved, and ancillary functions that compute the parametric models and provide this mesh with the required boundary conditions and body forces. The linking is made at the external surfaces of the rotor for the thermal variables, since considering a symmetrical machine, no heat is exchanged through its middle cross section. Conversely for the electrical ones, the connection between the models is done at the bars surfaces in that cross section and current insulation is assumed for the rest, either because they are surrounded by air or also due to symmetry in the shaft and iron. Two coupled FE solvers constitute the core of the combined model (Fig. 1). The first one, static current, is endowed with the task of calculating in the rotor the distribution of the currents, considered DC within the time step, and their corresponding joule heating $P_{\text {joule }}$ according to the potential at the middle of the bars $V_{b a r}$ supplied by the EM model, and the second one is used to calculate the heat transfer taking into account the temperature on the rotor external surfaces computed by the thermal network $\theta_{\text {ext }}$. To model the effect of the hot spot, the conductivity of aluminum $\sigma(\theta)$ has been considered temperature-dependent; therefore, this value is updated in the first solver at the end of the second's computation.

Routines written in FORTRAN and compiled as dll's are called before each time step by the FE software in order to recalculate the parametric models according to the previous FEM solution, using the rotor external temperatures $\theta_{\text {rotor }}$ and currents in bars $I_{b a r}$, and update the boundary conditions and body forces accordingly. The skin effect and the iron losses are modeled separately by other two subroutines and updated on the nodes of the mesh. Finally, a post processing FE linear elastic stage determines the mechanical effects caused by the heating.

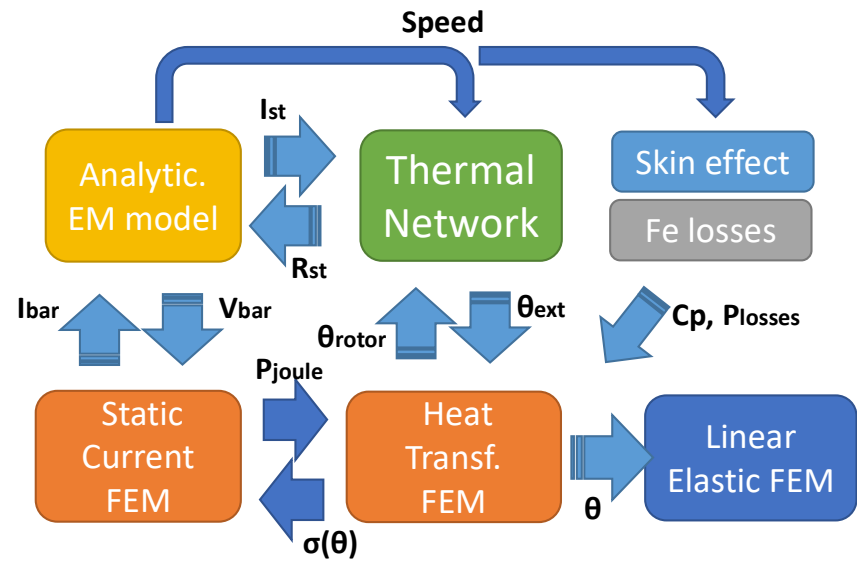

Fig. 1. Computational procedure comprising a FEM core (orange) responsible for obtaining the heating of the rotor during the transient, a post-processing FE module (right, dark blue) to calculate the mechanical effects of this heating and four ancillary functions modelling for each time step the thermal behavior of the stator (thermal network), the electromagnetic state of the motor (analytical EM model) and the losses and skin effect.

\section{A. FEM model}

The numerical computation tool used for solving partial differential equations in this work has been Elmer FEM, a software developed mainly by the CSC - IT Center for Science, Finland, since 1995 and released as open source in 2005 [24]. It includes more than 20 physical models and several numerical methods. Initially aimed at taking advantage of parallel computing resources, its modularity offers high flexibility when tackling complex multiphysics problems. For pre- and post-processing, commercial software and Paraview [25] were utilized instead.

The coupling of two FEM modules, heat transfer and static current conduction, allows solving in sequence the Maxwell's and heat equations in the rotor mesh for each time step. From a quasistatic point of view (skin effect is neglected here):

$$
\begin{gathered}
\nabla \cdot \vec{D}=\rho \\
\nabla \times \vec{E} \approx 0
\end{gathered}
$$




$$
\nabla \times \vec{H}=\vec{J}+\frac{\partial \vec{D}}{\partial t}
$$

The electric field can thus be expressed in terms of the electric scalar potential $\phi$ and taking into account the relation between current density and electric field in (3), it yields:

$$
\nabla \cdot \sigma(\theta) \nabla \phi=\frac{\partial \rho}{\partial t}
$$

$\sigma$ being the electric conductivity, which in the aluminum regions is variable with temperature [26] and provides the coupling parameter between the Static Current and Heat Transfer FE modules, whereas in the rotor's iron $\sigma$ is a second order constant tensor to take into account the negligible conductivity in the axial direction, being the values in the other two directions the corresponding to the interbar resistance assumed in [27]. Eq. (4) is used to solve the electric potential whilst the Joule heating is computed by:

$$
h_{\text {joule }}=\nabla \phi \cdot \sigma(\theta) \nabla \phi
$$

which constitutes, the internal generation input to the heat equation $h$ in the aluminum regions of the mesh, solved by the corresponding module:

$$
\rho_{d} c_{p}\left(\frac{\partial \theta}{\partial t}+(\vec{u} \cdot \nabla \theta)\right)-\nabla \cdot(k \nabla \theta)=\rho_{d} h
$$

$\rho_{d}$ being in this case the density of the material, $c_{p}$ the heat capacity and $k$ a second order tensor accounting for the thermal conductivity of the material, which is heavily anisotropic in the iron regions due to the effect of the laminations [1]. In this area of the rotor the generation term is also modified to take into account the Eddy current losses as:

$$
h=h_{\text {joule }}+h_{\text {losses }}
$$

Decoupled from this FE computation core devised to obtain the DC current and heat distribution in the rotor, the mechanical effects in the cage produced by the heating are calculated as a post-processing stage by the Elmer's Linear Elastic module, using the dynamical equation for elastic deformation of solids (8) to obtain the displacements in mesh:

$$
\rho_{d} \frac{\partial^{2} \vec{d}}{\partial t^{2}}-\nabla \cdot \tau=\vec{f}
$$

$\vec{d}$ being the displacement field, $\vec{f}$ a volume force, and $\tau$ the stress tensor:

$$
\tau^{i j}=C(\theta)^{i j k l} \varepsilon_{k l}-\beta^{i j}\left(\theta-\theta_{0}\right)
$$

where $C(\theta)$ is elastic modulus tensor (unlike [28] dependent on the actual temperature of the rotor nodes $\theta$ at the time step considered [29]), $\beta$ the thermal expansion tensor, in this particular case both reduced to scalars and $\theta_{0}$ the reference temperature. The linear strain is obtained simply as:

$$
\varepsilon=\frac{1}{2}\left(\nabla \vec{d}+(\nabla \vec{d})^{T}\right)
$$

Since no magnetic behavior is taken into account in these partial differential equations, the feeding of the rotor bars constitutes a non-trivial question. According to a previous analytical study [27], due to the ring connections at both ends of the bar, the maximum interbar voltage was achieved around the middle point of this element. Hence in order to provide a suitable boundary to impose the electrical potential $\phi$ obtained from the EM routine and to reduce the number of degrees of freedom of the model, the mesh solved by these three Elmer modules depicts just half of the rotor (Fig. 2).

\section{B. Analytical models}

Before each time step, the functions below are called by the corresponding solvers to update several parameters in the mesh.

\section{1) Thermal Network:}

A transient lumped thermal network was formed to study the impact of rotor bar breakage on the machine's stator side. This network offers an analytical solution of the transient heat conduction across the machine's stator, as well as the associated convection processes. Such a lumped model is a simple way to observe the time-dependent temperature distribution in the stator. It is governed by equation:

$$
\left[C_{e}\right] \frac{d[\theta]}{d t}+[G] \cdot[\theta]=[P]
$$

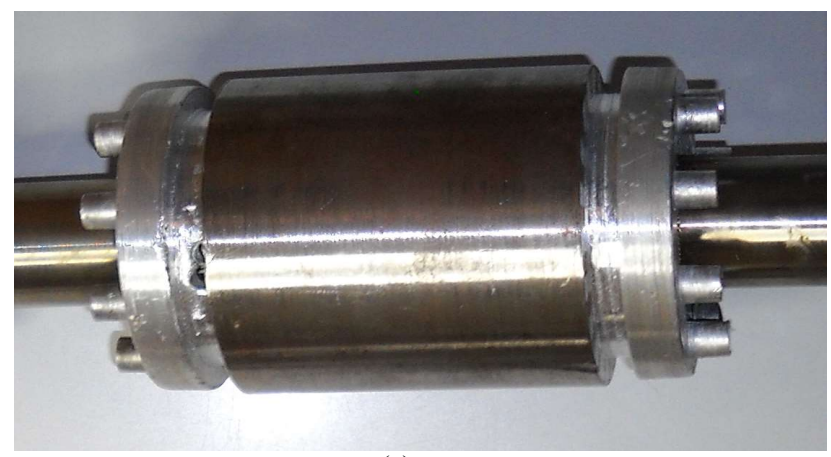

(a)

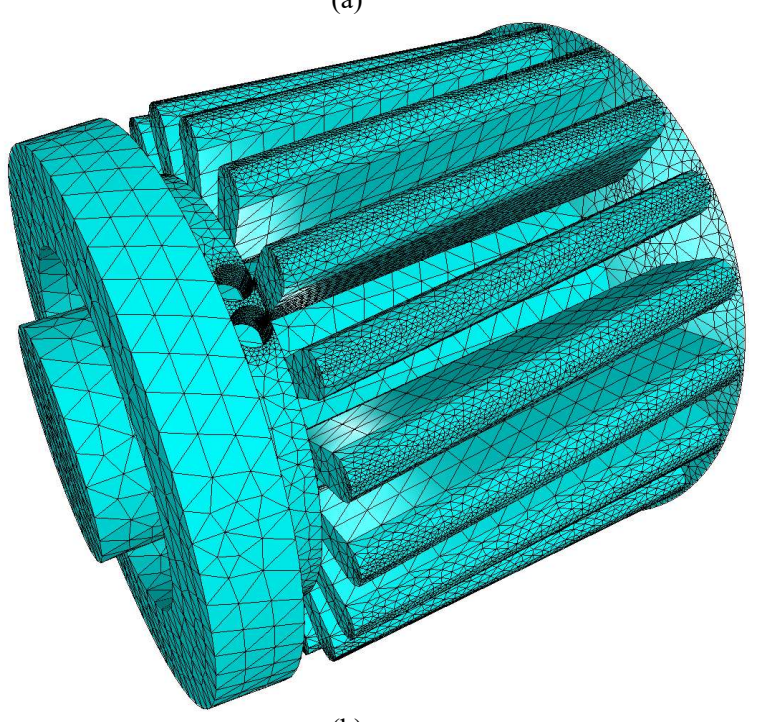

(b)

Fig. 2. a) Rotor at the end of the fatigue test carried out in [18] and b) mesh representing half of it during the final stage of that same test (the rotor's iron has been removed to show the bars). 
where, $C_{e}$ is thermal capacity of each node, $[G]$ matrix of thermal conductances and $P$ is the power generation at the nodes. This transient lumped model neglects possible temperature gradient within a lumped solid during the transient process.

The corresponding thermal network shown in figure Fig. 3 encompasses just half of the stator due to symmetry. Since the machine is totally enclosed, cooling occurs through forced convection over machine's outer frame, represented by Rol. Rs 1 accounts for the frame-yoke constant resistance. As indicated in [1], two T circuits model the yoke and teeth (nodes 12 and 13), with the small negative resistances $R s 3$ and $R s 5$ necessary to adapt a distributed source into a point heat injection. Rs6, $R s 8$ and $R s 9$ constitute another $\mathrm{T}$ circuit accounting for the longitudinal temperature variation in the slot windings and similarly $R s 10, R s 12, R s 14$ and $R s 15$ for the end windings, being $R s 8$ and $R s 14$ the conductors insulation thermal resistance and $R s 15$ accounting for the convection to the end space. The bearing ( $R b 1-R b 3$ for the rolling elements and both rings) and the end cap ( $R d f)$ have also been included in the network. Nodes numbered 4, 5, 8 bridge the stator and rotor and receive the power losses $q$ from the corresponding boundary conditions on the rotor side (end ring, rotor iron and shaft), as indicated by the arrows, whereas their temperatures $\theta_{\text {ext }}$ update the gradient value in the Neumann boundary condition of the mesh for the next time step $t$ :

$$
k\left(\theta^{t}-\theta_{\text {ext }}^{t-1}\right)=q^{t}
$$

$k$ here stands for the convection coefficient on the surfaces of rotor and end ring and the conduction coefficient on the shaft. In this way both models are coupled. The end ring convection heat transfer coefficient has been considered speed-dependent during the simulation.

\section{2) Analytical Model of the Motor:}

For thermally loading the motor in this work an improvement of the solution devised in [17] was preferred. Instead of a per-phase model of the machine, the analytical model published in [22] was specifically devised to reproduce the effect of several faults, including electrical asymmetries, thus this model takes into account the position and current of every conductor along the airgap. In addition, a circular convolution computed by means of the FFT allows a precise calculation of self and mutual inductances. This process is carried out in MATLAB for the motor whose characteristics are shown in the Appendix before the actual simulation begins, filling a 3D lookup table, which is read by the FORTRAN routine when the FEM simulation starts and stored in the computer's memory for the rest of its duration.

Given the different time constants involved in thermal and electromagnetic phenomena, this analytical model operates at 1,000 times the rate of the FE one (1,000 time steps of the EM model are computed when the FE model request updating the boundary conditions at a new time). As in the previous point, the ordinary differential equations for each circuit are integrated using the Crank-Nicolson method. Half the resistive part $R_{r} I_{r}$ of the voltage equation (13) is applied to the corresponding bar:

$$
[0]=\left[R_{r}\right] \cdot\left[I_{r}\right]+\frac{d\left[\psi_{r}\right]}{d t}
$$

The integration of the current circulating through the same boundary where the voltage is applied is yielded by the FE solver and used to update the bar resistance in the EM model through a low-pass filter.

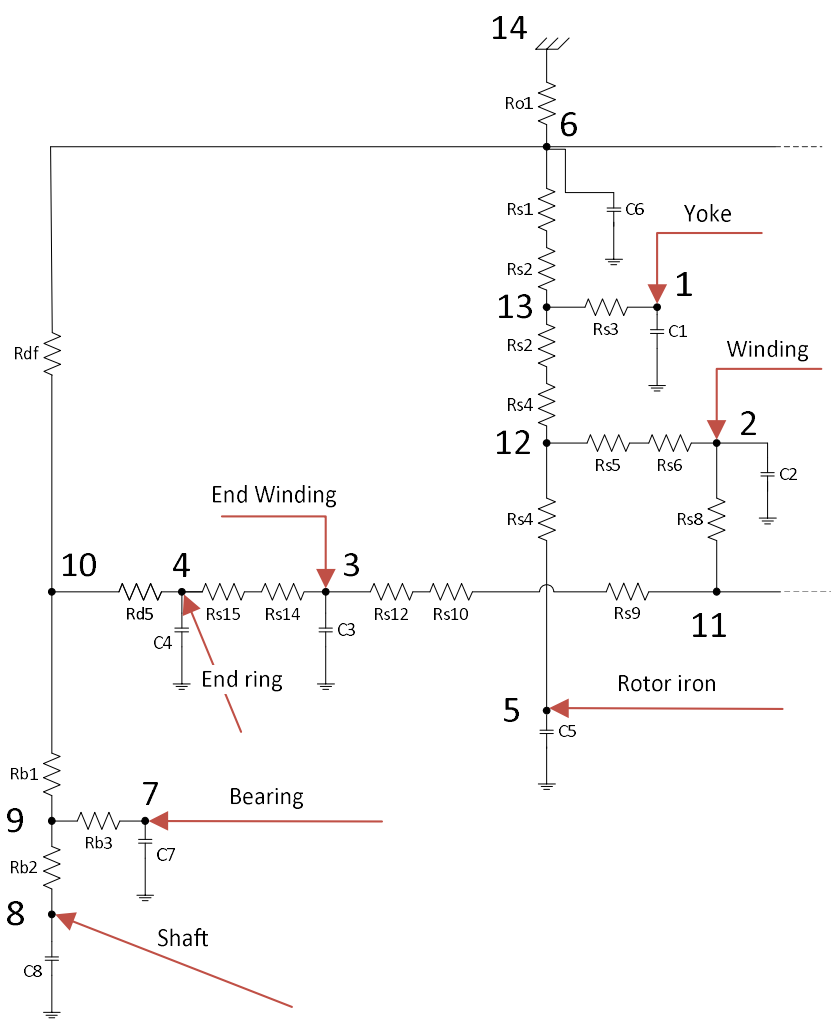

Fig. 3. Thermal network used to model the thermal behavior of the stator. The power obtained from the temperature difference on the boundary conditions of the FE model is injected in nodes 4, 5, 8 .

\section{3) Losses and Skin Effect Modelling:}

Elmer provides the capacity of imposing values on the nodes for the parameters used in the computation. This has an immediate application for adding the iron losses to the model by computing a further generation term (7) for the heat equation (6). An iron losses model that takes into account their variation with frequency is used for this purpose [23]. The magnetic flux density fundamental frequency in the rotor $f_{s}$ is obtained from the movement equations of the motor, which are integrated along the electromagnetic ones in the previous module, whereas the peak magnitude is assumed, according to previous $2 \mathrm{D} \mathrm{FE}$ simulations of the same motor, constant with a value of 1.8 T. The corresponding values for M43 electric steel at that flux density are used in this work for all four coefficients in (14). Nevertheless, the hysteresis coefficient $k_{h}$ and the exponent $\alpha$ are also implemented in the code as frequency dependent, as indicated in [23].

$$
\frac{h_{\text {losses }}}{m_{\text {iron }}}=k_{h}\left(f_{s}\right) f B^{\alpha\left(f_{s}\right)}+k_{e} f_{s}^{2} B^{2}+k_{a} f_{s}^{1.5} B^{1.5}
$$


Since the aim of the model is to compute the heating of the rotor during transients an adequate calculation of the skin effect is necessary; however, no magnetic variables are solved in the mesh; therefore the strategy followed in this work has been to reflect the differential heating it causes on the bars by modifying the heat capacity of the bar along its height, according to the rotational speed supplied by the EM analytical model. The analytical equations used to compute it assumed a sinusoidal variation of the current $[1,16]$. The curve obtained for each frequency was normalized to 1 in order to avoid altering the overall heat capacity of the rotor. A polynomial-formulated surface was fitted to the resulting points (Fig. 4). No skin effect was considered whatsoever in the end ring.

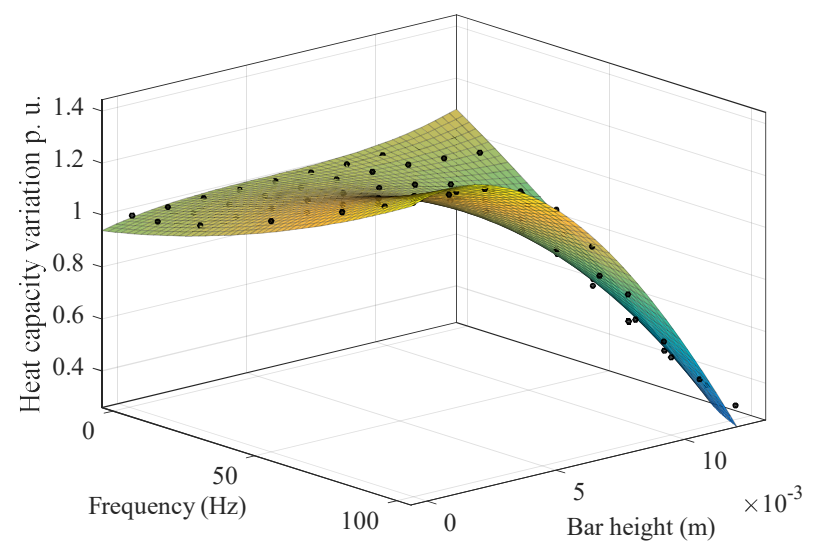

Fig. 4. Variation of the aluminum heat capacity along the height of the bar for frequencies $1-100 \mathrm{~Hz}$.

\section{INITIAL VALIDATION (COLD STARTUP)}

With the objective of carrying out an initial assessment of the model's performance a cold startup simulation is performed. For this transient, accurate initial conditions of the motor's temperatures can be established and hence an easier comparison with the data provided by a thermocouple, installed during the experiments through the connection box on the stator and next to end windings, is possible. This would reflect the electrical and thermal accuracy of the model.

The parameters and material's characteristics used for both simulations are presented in Table I:

\section{A. Assumptions}

With the aim of critically establishing the utility of the model proposed in this work, the assumptions made on all its modules are enumerated and discussed:

1. No saturation is considered in any of the models. This prevents the accurate linking of the effect of the fault on the stator currents.

2. A current distribution according to a DC excitation is computed. Electrically, only the effect of the resistance is taken into account in the mesh.

3. The skin effect in the end ring is neglected. Despite the small height of the hot spot, at the relatively high frequencies experienced during braking (100-50 Hz) this might cause particularly high temperatures on its upper surface.

4. Skin effect and iron losses, when modelled, are done so according to a sinusoidal variation of flux density assumption. Slotting harmonics cause additional losses next to the surface of the rotor not accounted for.

5. Heat convection is not computed. Analytical equations are used instead to obtain global heat transfer coefficients that are applied to whole surfaces: shaft, end ring and rotor iron, thus averaging those heat fluxes.

6. Thermal contact resistances, a big source of uncertainty in thermal studies, are also ignored.

7. No heat transfer by radiation is considered. Although this effect would slightly lower the temperature at the bottom of the hot spot, facing the shaft, the heat transfer by radiation in the rest of directions is low, due to similar temperatures of the receiving surfaces (end ring, end winding).

8. In the linear elastic analysis, the influence of iron is not taken into account.

TABLE I

Parameters of the Startup and Plug Stopping Simulations

\begin{tabular}{|c|c|c|c|}
\hline Voltage & $230 \mathrm{~V}$ & $k_{z}(\mathbf{F e})$ & $0.6 \mathrm{~W} / \mathrm{m} \cdot \mathrm{K}$ \\
\hline Frequency & $50 \mathrm{~Hz}$ & $k$ (shaft) & $31 \mathrm{~W} / \mathrm{m} \cdot \mathrm{K}$ \\
\hline Inertia, $J$ & $0.13 \mathrm{~kg} \cdot \mathrm{m}^{2}$ & $C p$ (Fe, shaft) & $449 \mathrm{~J} / \mathrm{kg} \mathrm{K}$ \\
\hline $\begin{array}{c}\text { External } \\
\text { temperature }\end{array}$ & $298 \mathrm{~K}$ & $\boldsymbol{R}_{\text {stator }}$ (init) & $2 \Omega$ \\
\hline $\begin{array}{c}\text { Time step, EM } \\
\text { model }\end{array}$ & $1 \cdot 10^{-6} \mathrm{~s}$ & $\Delta R / R(\mathrm{Cu})$ & $4.2910^{-3} \mathrm{~K}^{-1}$ \\
\hline $\begin{array}{c}\text { Time step, FE } \\
\text { model }\end{array}$ & $1 \cdot 10^{-3} \mathrm{~s}$ & $\boldsymbol{R}_{b a r}$ (init) & $2.1510^{-4} \Omega$ \\
\hline$C p(\mathrm{Al})$ & $897 \mathrm{~J} / \mathrm{kg} \mathrm{K}$ & $\boldsymbol{R}_{b a r, h s}$ (init) & $3.0510^{-4} \Omega$ \\
\hline$\sigma_{x, y}(\mathrm{Fe})$ & $1.6710^{3} \mathrm{~S}$ & $\theta_{0}$ & $317 \mathrm{~K}, 443 \mathrm{~K}$ \\
\hline$\sigma_{z}(\mathbf{F e})$ & 0 & $v(\mathrm{Al})$ & 0.334 \\
\hline $\boldsymbol{k}_{x, y}(\mathbf{F e})$ & $31 \mathrm{~W} / \mathrm{m} \cdot \mathrm{K}$ & $\beta$ & $23.1 \cdot 10^{-6}$ \\
\hline
\end{tabular}

\section{B. Cold startup results}

During the periods the fatigue test carried out in [18] was periodically stopped the motor studied almost reached ambient temperature. This allows establishing a constant distribution to initialize the mesh and the thermal network and thus an easier assessment of the electrical and thermal models performance, contrary to the plug stopping transient, which was carried out after 20 seconds of operation.

Fig. 5 shows the values recorded by the thermocouple installed in the motor's connection box for 5 cold startups during the last part of the fatigue test when the bar breakage developed. The difference between the earlier and the later group agrees with a slight variation of ambient temperature $(0.4 \mathrm{~K})$ as the test progressed.

In addition, simulated temperatures up to 7 seconds, when the startup transient ends, for the end space area (nodes 3, 4 and 10 in Fig. 3) and the outer part of the stator (node 13), are also depicted. The recorded values don't correspond to any of them, as it is expected from the position of the sensor 
and the parametrization applied in the synthesis of the thermal network. However, the thermal inertia showed in the evolution corresponds to the stator yoke and the motor's outer surfaces, as well as a total temperature increase influenced by the end space region.

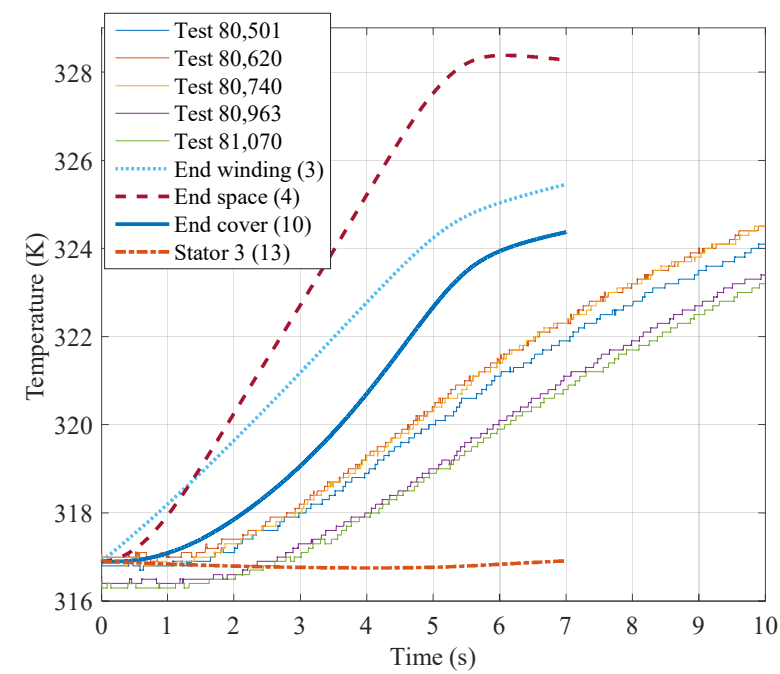

Fig. 5. Comparison of the temperature rise recorded by the thermocouple during five experimental cold startups and the temperature rise on several nodes computed by the model for the same transient.

\section{Results (Plug StOPPING)}

The most demanding conditions the motor suffered during the fatigue test in [18] where achieved at the end of each cycle, during the plug stopping. In order to have an insight of the mechanical effects this transient caused another simulation is carried out. The initial condition is in this case warm rotor $(443 \mathrm{~K})$ being the FE model initialized with this value for the whole mesh whereas for initializing the thermal network a full version, also including the rotor, was run following the cycling of [18] until the rotor iron values reached that temperature. The other parameters of the simulation are shown in Table I.

\section{A. Electrical results}

The hot spot has a small influence in the bar currents measured at the middle of the rotor, as shown in Fig. 6, where the standard deviation of this magnitude for each bar during the transient is compared. A decrease of just $2.2 \%$ is appreciated for the bar facing the hot spot, whereas the neighboring ones are overloaded, 1.4\% (Bar 2) and $0.34 \%$ (Bar 20). Furthermore, it can also be seen that both models work with overall similar values of current.

In addition, the resistance values for the rotor bars that the Electromagnetic analytical model utilizes during the simulation are depicted in Fig. 7. The initial resistance difference introduced by the saddle is $41.9 \%$. The feedback loop that updates these values according to the currents measured in the middle section of the rotor is activated 100 ms after connection to avoid sharp variations due to the electromagnetic transient that may destabilize the EM model. At the end of the plug stopping, the bar facing the hot spot has an observed resistance $14.1 \%$ greater, whereas the others have just increased around 5\%. As expected, Bar 20 (Fig. 6, a2) and Bar 2 (Fig. 6, a3) suffer the effect of their higher load. The spread of the rest is a consequence on the different heating caused by the asymmetric components of the electromagnetic transient.

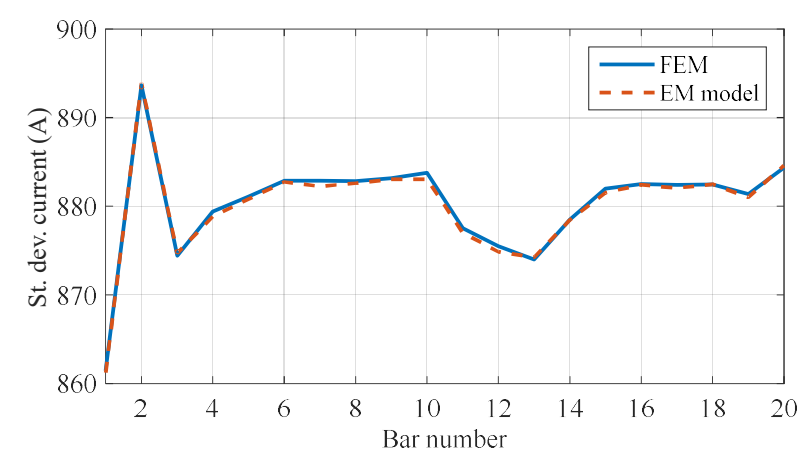

Fig. 6. Standard deviation of the currents in the bars at the middle of the rotor during the simulation. Bar 1 is facing the hot spot and hence shows an increased resistance. Both FE and EM models work with similar values.

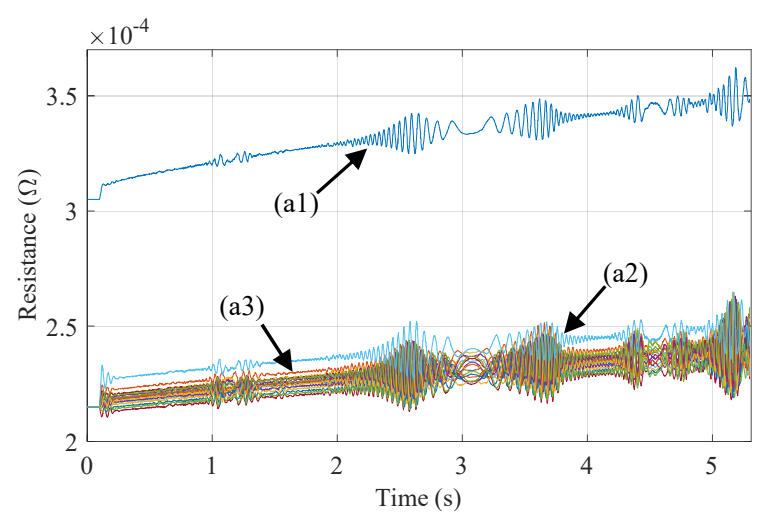

Fig. 7. Evolution of bar resistances during the transient as processed by the EM model. The highest values are observed on Bar 1 (a1), Bar 20 (a2) and Bar 2 (a3).

Fig 8 shows the analysis of the stator currents using a time-frequency decomposition method based on the WignerVille distribution [18]. The low sideband harmonic (LSH-50, $(1-2 s) f$ ) generally used for diagnosing rotor asymmetries is clearly detected in case a); evolving from $150 \mathrm{~Hz}$ to $50 \mathrm{~Hz}$ (b1) as the slip progresses from 2 to 1 . However, when the material around the hot spot is defined as aluminum, instead of air, and the same simulation is performed, the component cannot be appreciated (Fig. 8 (b)). In both cases, the saliency harmonics reflect the decreasing speed of the rotor (b2).

\section{B. Thermal results}

Fig. 9 presents the main heat fluxes in and out the thermal network depicted in Fig. 3. The effect of the speed-dependent convection coefficients on nodes 4 and 6 is shown, reducing the heat transferred out of the end ring and stator. Yet the airgap-related 5, predominantly laminar, remains constant

The iron losses in the rotor at the beginning of the braking account for just $82 \mathrm{~W}(13.7 \mathrm{~W} / \mathrm{kg})$ compared to the $2.4 \mathrm{~kW}$ in resistive losses. 


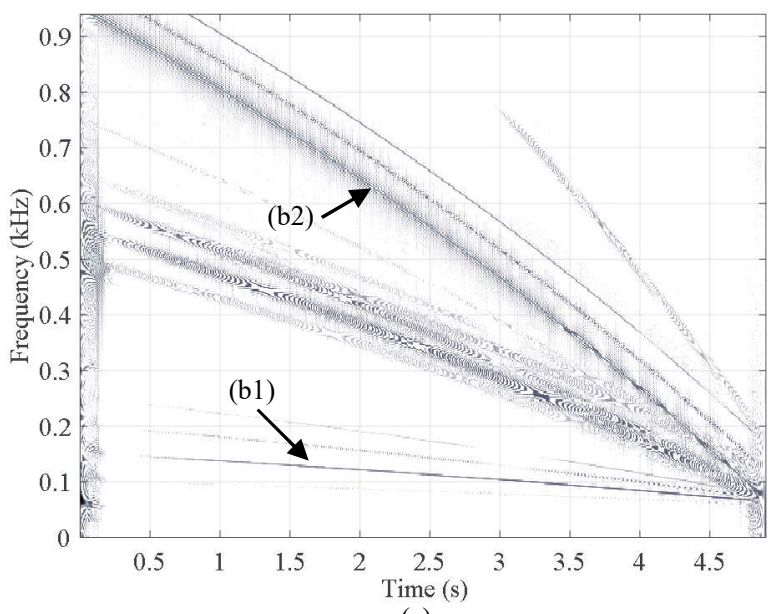

(a)

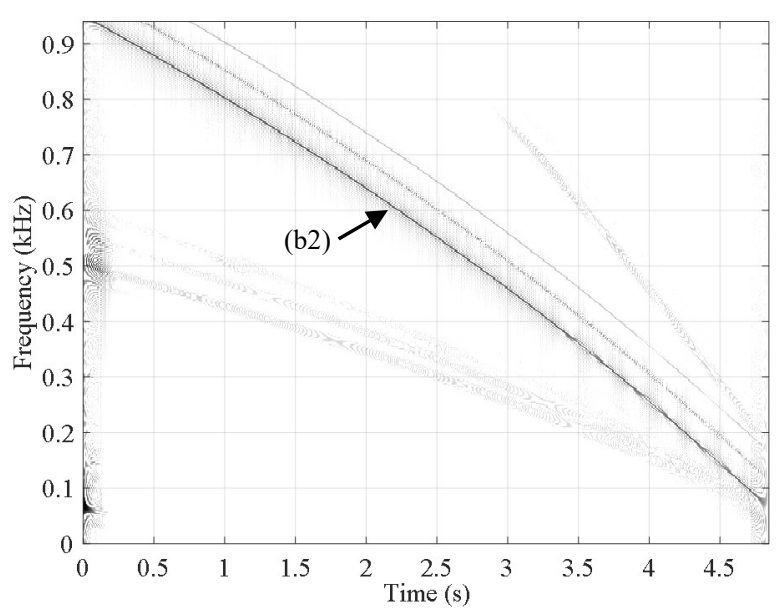

(b)

Fig. 8. a) Time-frequency analysis of the stator current, rotor having a hot spot. The rotor asymmetry component $(1-2 s) f(\mathrm{~b} 1)$ can be appreciated evolving form $150 \mathrm{~Hz}$ to $50 \mathrm{~Hz}$ during the transient, in the case of a healthy rotor $\mathrm{b})$, this component is not shown.

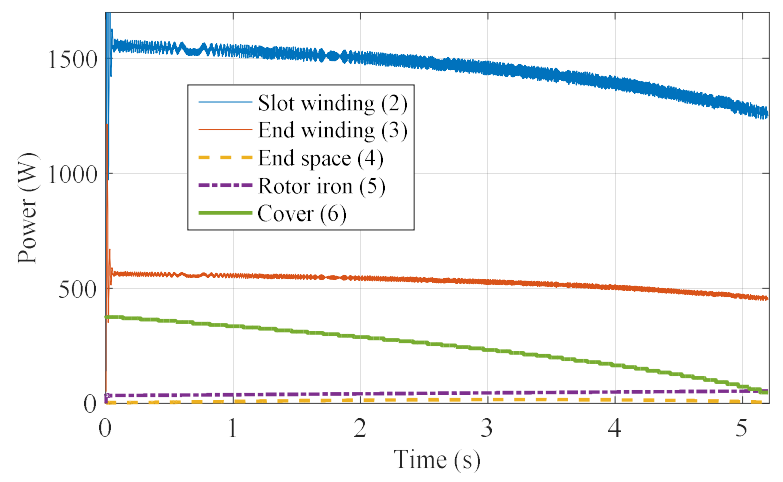

Fig. 9. Heat flux injected in nodes 2-5 and output through 6. The effect of the variable convection coefficients on 4 and 6 is appreciated.

Fig. 10 presents the temperatures in several nodes of the thermal network. The cycling has risen the values in the stator, where a high gradient develops at the end of the stationary operation and beginning of the braking. The temperatures of the most external nodes don't suffer a high increase during the transient, as experimentally verified, due to heat capacity of all the elements and the effect of the fan. The recorded increase measured by the thermocouple for Test 81,060 during this transient was $11 \mathrm{~K}$, whereas in the simulation the end space temperature (node 4) rose $11.3 \mathrm{~K}$ and the stator 3 (node 13) $3.6 \mathrm{~K}$.

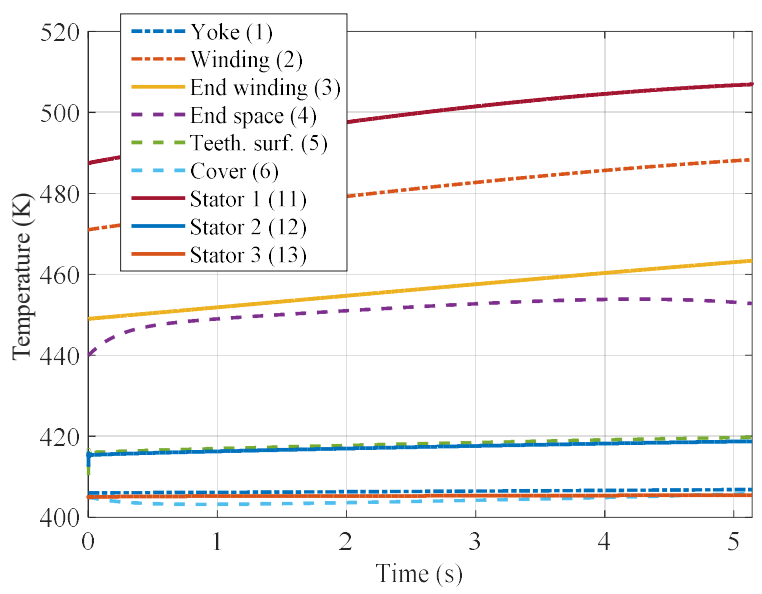

Fig. 10. Simulated temperatures in the stator nodes.

Finally, Fig. 11 (a) shows the temperatures reached in the rotor after five seconds of the braking simulation, which took around 100 hours in a PC, when the rotor was approaching standstill. The highest temperatures are accounted for at the saddle point at the end of the damaged bar, with a value of 90 $\mathrm{K}$ above the shaft. The low heat conductivity of the iron sheets in the axial direction prevents much of the heat produced in the end ring to be transferred elsewhere.

Fig. 11 (b) presents the section of the bars next to the symmetry plane in the middle of the rotor after 0.5 seconds of simulation, showing temperature difference within each one and among the one facing the hot spot and the overloaded, due to interbar currents, neighboring ones.

\section{Mechanical results}

Finally, applying the linear elastic solver the deformation and stress of the rotor due to the heating can be obtained (Fig. 12). Since no clearance was allowed between the bars and the iron and the former were casted in closed slots, high stresses were initially obtained on them. However, if only the cage is considered in this analysis, the hot spot becomes the highest stress area, being the Von Mises value of this magnitude $38.6 \mathrm{MPa}$, well above the yield tension of aluminum at that temperature $(14 \mathrm{MPa})$ thus causing plastic deformation. This permanent deformation, when the cage cools down, creates tensile stresses that initiate and propagate fatigue cracks. Maximum shear stresses are obtained at the same place where the crack developed in [18] (Fig. 12). The surrounding end ring material suffers lesser permanent effects, since both stress and temperature (Fig. 11 (a)) are lower, and hence the yield limit of aluminum (17 $\mathrm{MPa}$ at $500 \mathrm{~K}$ ) is barely reached. 


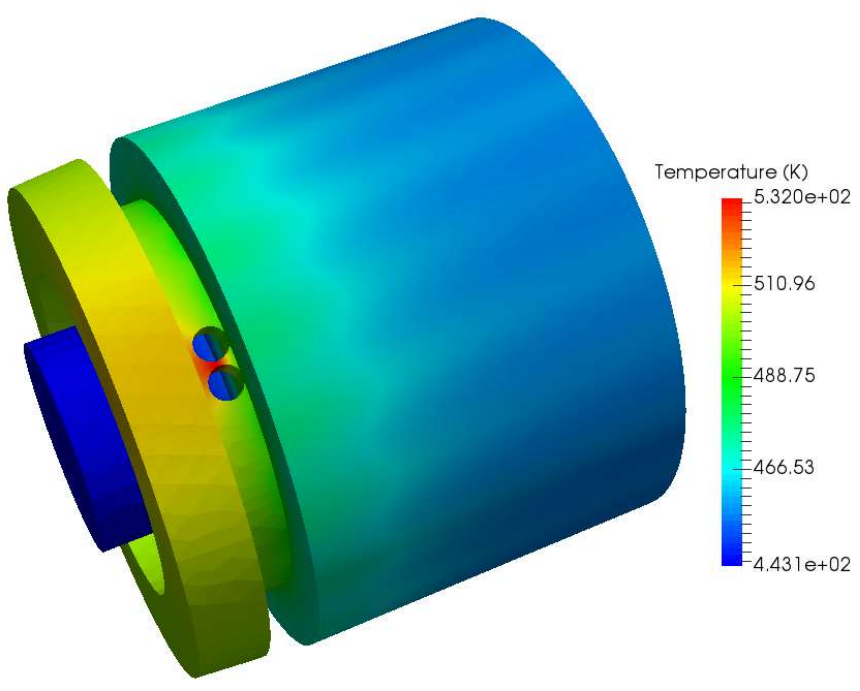

(a)

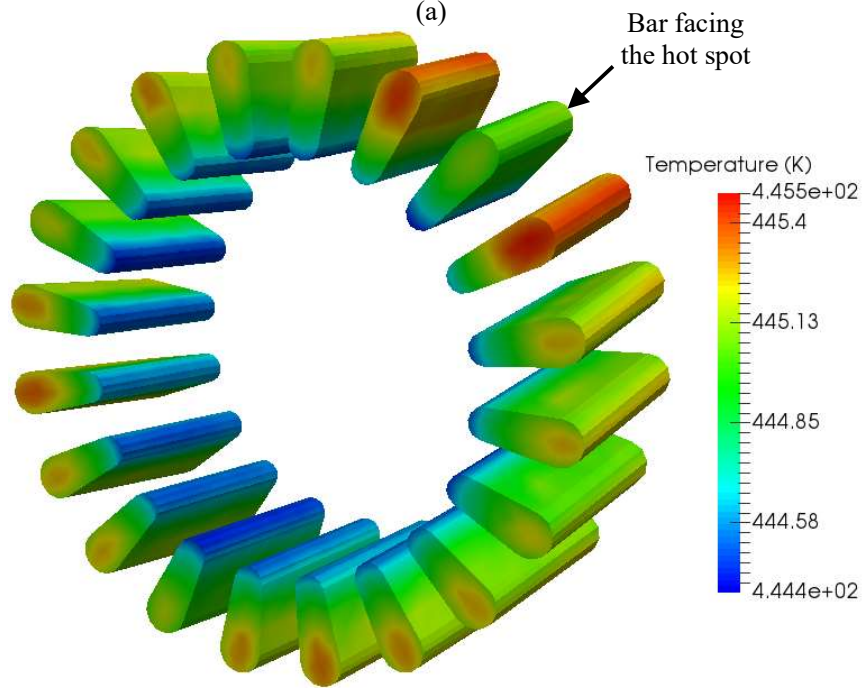

(b)

Fig. 11. a) Temperature distribution in the half rotor at the end of the plug stopping transient (warm rotor, initial temperature $443 \mathrm{~K}$ ), and b) temperature in the bars after 0.5 seconds.

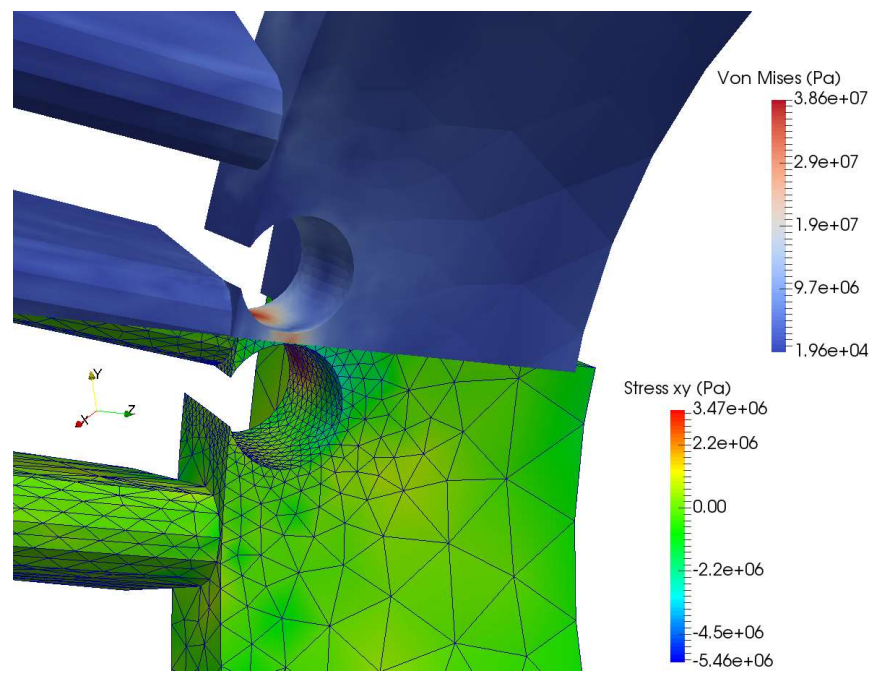

Fig. 12. Von Mises stress, shear stress ( $x y$-plane) and deformation (x50) on the hot spot due to thermal expansion at the end of the plug stopping simulation (5 seconds).

\section{CONCLUSIONS}

This work presents an approach intended to provide an insight on the effects of a hot spot in a rotor cage during transient operation. To reduce the computational requirements of such complex problem, a combined analytical-FE simulation is proposed, the EM and the Joule heating phenomena have been segregated in two models, and the thermal behavior of the stator has also been implemented in a separate lumped parameter thermal model. Several assumptions have been made, however, initial results shows the capability of the approach for linking the size of the defect with its electrical, thermal and mechanical effects, among others, the appearance of temperature gradients in the rotor cage due to the limited heat transfer capacity of the surrounding material and the corresponding stresses beyond the yield limit in certain areas of the hot spot, which qualitatively explains the crack propagation's behavior observed in a previous fatigue test.

Future works will be focused on implementing a fatigue model based on these results for application in the RUL estimation and increasing the accuracy of the approach. For instance, taking into account the effect of saturation in the EM analysis would allow linking cause (the size of the hot spot) and the effect produced in the stator currents. This could be carried out either by improving the analytical model or coupling a traditional 2D FE motor simulation. The thermal part can be enhanced by performing short EM 3D simulations to ascertain the current distribution in the cage at different frequencies and carrying out the corresponding quantitative validations by experimental procedures.

\section{APPENDIX}

Motor characteristics: Star connected, rated voltage $\left(\mathrm{U}_{\mathrm{n}}\right)$ : $400 \mathrm{~V}$, rated power $\left(\mathrm{P}_{\mathrm{n}}\right): 1.5 \mathrm{~kW}, 1$ pole pairs, stator rated current $\left(\mathrm{I}_{1 \mathrm{n}}\right): 3.25 \mathrm{~A}$, rated speed $\left(\mathrm{n}_{\mathrm{n}}\right): 2860 \mathrm{r} / \mathrm{min}$.

\section{REFERENCES}

[1] Design of Rotating Electrical Machines, J. Pyrhönen, T. Jokinen, V. Hrabovcová, Edt. Wiley, second edition, 2014.

[2] A. Boglietti, A. Cavagnino, M. Lazzari, M. Pastorelli, "A simplified thermal model for variable-speed self-cooled industrial induction motor," IEEE Trans. Ind. Appl., vol. 39, no. 4, pp. 945 - 952, Jul.-Aug. 2003.

[3] DIN EN IEC 60034-6. Rotating electrical machines. Part 6: methods of cooling.

[4] J. Fouladgar; E. Chauveau, "The influence of the harmonics on the temperature of electrical Machines," IEEE Trans. Magn., vol. 41, no. 5, pp. 1644-1647, May 2005.

[5] M. Polikarpova et Al. "Hybrid Cooling Method of Axial-Flux Permanent-Magnet Machines for Vehicle Applications," IEEE Trans. Ind. Electron., Vol. 62, no. 12, pp. 7382 - 7390, Dec. 2015.

[6] V. T. Buyukdegirmenci, P. T. Krein, "Induction Machine Characterization for Short-Term or Momentary Stall Torque," IEEE Trans. Ind. Appl., vol. 51, no. 3, pp. 2237-2245, May-Jun. 2015,

[7] S. Jurkovic, K. M. Rahman, J. C. Morgante, P. J. Savagian, "Induction Machine Design and Analysis for General Motors e-Assist Electrification Technology," IEEE Trans. Ind. Appl., vol. 51, no. 1, pp. $631-639$, Jan.-Feb. 2015.

[8] N. Nisar, Structural Optimization and Thermal Modeling of Flux Switching Machine, Master's thesis dissertation, Aalto University, 2015. 
[9] M. Riera-Guasp, J. A. Antonino-Daviu, G.-A. Capolino, “Advances in Electrical Machine, Power Electronic, and Drive Condition Monitoring and Fault Detection: State of the Art," IEEE Trans. Ind. Electron., Vol. 62, no. 3, pp. 1746 - 1759, Mar. 2015.

[10] C. Ly, K. Tom, C. S. Byington, R. Patrick, G. J. Vachtsevanos, "Fault diagnosis and failure prognosis for engineering systems: A global perspective automation science and engineering," in Proc. CASE, 2009, pp. 108-115, Bangalore, India.

[11] V. Peesapati, R. Gardner, R. Lowndes, I. Cotton, B. Twomey, L. Dunsby, R. Balcombe, "Impact of thermal cycling on high voltage coils used in marine generators using FEA methods," IEEE Electrical Insulation Conference (EIC), Seattle, WA, Jun. 7-10 2015, pp. 434-43.

[12] A. Bellini, F. Filippetti, C. Tassoni, G.-A. Capolino, "Advances in diagnostic techniques for induction machines," IEEE Trans. Ind. Electron., Vol. 55, no. 12, pp. 4109-4126, Dec., 2008.

[13] M. Hodowanec, W. R. Finley, Copper Versus Aluminum-Which Construction Is Best? [Induction Motor Rotors], IEEE Ind. Appl. Magazine, vol. 8, 2002, pp. 14-25.

[14] R. Yabiku, R. Fialho, L. Teran, A. Santos, E. Rangel, D. Dutra, "A comparative study between copper and aluminum induction squirrel cage constructions," in Proc. Petroleum and Chemical Industry Conference (PCIC), San Antonio, TX, USA, Sept. 20-22 2010, pp. 1-9.

[15] M. F. Cabanas, J. L. Ruiz Gonzalez, J. L. B. Sampayo, M. G. Melero, C. H. Rojas, F. Pedrayes, A. Arguelles, J. Vina, "Analysis of the fatigue causes on the rotor bars of squirrel cage asynchronous motors: Experimental analysis and modelling of medium voltage motors," in Proc. SDEMPED, 2003, Stone Mountain, GA, USA, pp. 247-252.

[16] C. D.Pitis, "Thermo-mechanical stresses of the squirrel cage rotors in adverse load conditions," in Proc. ISEI, 2008, pp. 579-585, Vancouver, BC, Canada.

[17] R. Yabiku, R. Fialho, L. Teran, M. E. Ramos, N. Kawasaki, "Use of thermal network on determining the temperature distribution inside electric motors in steady-state and dynamic conditions," IEEE Trans. Ind. Appl., vol. 46, no. 5, pp. 1787-1795, Sep./Oct., 2010.

[18] V. Climente-Alarcon, J. A. Antonino-Daviu, E.G. Strangas, M. RieraGuasp, "Rotor-Bar Breakage Mechanism and Prognosis in an Induction Motor," IEEE Trans. Ind. Electron., vol. 62, no. 3, pp. 1814-1825, Mar. 2015.
[19] M. S. Rajagopal, K. N. Seetharamu, P. A. Ashwathnarayana, "Transient thermal analysis of induction motors," IEEE Trans. Energy Convers., vol. 13, no. 1, pp. 62 - 69, Mar. 1998.

[20] P. H. Mellor, D. Roberts, D. R. Turner, "Lumped parameter thermal model for electrical machines of TEFC design," IEE Proceedings B Electric Power Applications, vol. 138, no. 5, pp. 205 - 218, Sept. 1991.

[21] A. Boglietti, A. Cavagnino, D. Staton, M .Shanel, M. Mueller, C. Mejuto, "Evolution and Modern Approaches for Thermal Analysis of Electrical Machines," IEEE Trans. Ind. Electron., vol. 56, no. 3, pp. 871-882, Mar. 2009.

[22] M. Pineda-Sanchez, V. Climente-Alarcon, R. Riera-Guasp, R. PuchePanadero, J. Pons-Llinares, "Enhanced Simulink induction motor model for education and maintenance training," Journal of Systemics, Cybernetics and Informatics, vol. 10, no. 2, 2012.

[23] D. M. Ionel, M. Popescu, S. J. Dellinger, T. J. E. Miller, R. J. Heideman, M. I. McGilp, "On the variation with flux and frequency of the core loss coefficients in electrical machines," IEEE Trans. Ind. Appl., Vol. 42, No. 3, May-June 2006, pp. $658-667$.

[24] Elmer Finite Element Software, CSC - IT Center for Science, FINLAND, [online], Available: https://www.csc.fi/web/elmer

[25] Ayachit, U., "The ParaView Guide: A Parallel Visualization Application," Kitware, ISBN 978-1930934306, 2015.

[26] P. D. Desai, H. M. James, C. Y. Ho, "Electrical Resistivity of Aluminum and Manganese," J. Phys. Chem. Ref. Data, vol. 13, no. 4, 1984.

[27] N. Christofides, "Origins of load losses in induction motors with cast aluminium rotors," Proc. of the Institution of Electrical Engineers, vol. 112, no. 12, pp. $2317-2332,1965$.

[28] V. Climente-Alarcon, D. Nair, R. Sundaria, J. Antonino-Daviu, A. Arkkio, "Combined Model for Simulating the Effect of a Heavy Transient on a Damaged Rotor Cage", Proc. of ICEM 2016, Lausanne, Switzerland, Sept. 4-7, 2016.

[29] E. Velasco, R. Colás, S. Valtierra, J. F. Mojica, A model for thermal fatigue in aluminium casting alloy, Int. J. Fatigue, Vol. 17, No. 6, 1995, pp. 399-406. 